\title{
Screening and Authentication of Key Genes and Prognostic Value Analysis in Oligodendroglial Tumors
}

\section{Zhonghua Lv}

Tumor Hospital of Harbin Medical University

\section{Bongbo Bao}

Tumor Hospital of Harbin Medical University

Peng Liang ( $\sim$ liangpenghmu@hotmail.com )

Tumor Hospital of Harbin Medical University https://orcid.org/0000-0002-9394-5016

\section{Research}

Keywords: Bioinformatics, oligodendroglial tumor, Oligodendroglioma, Anaplastic oligodendroglioma, Differentially expressed genes, Prognosis

Posted Date: June 1st, 2020

DOl: https://doi.org/10.21203/rs.3.rs-31293/v1

License: (c) (1) This work is licensed under a Creative Commons Attribution 4.0 International License. Read Full License 


\section{Abstract}

Background: Emerging evidence indicates that various functional genes with altered expression are involved in the human tumor progression. This study is aimed at identifying novel key genes that may be used for oligodendroglial tumor diagnosis, prognosis, and targeted therapy.

Methods: This study included three expression profiles (GSE15824, GSE29796 and GSE108474) obtained from the Gene Expression Omnibus (GEO). GEO2R was used to analyze the differentially expressed genes (DEGs) between normal samples and oligodendroglial tumor, including oligodendroglioma and anaplastic oligodendroglioma. The functional and pathway enrichment analysis was performed by the Database for Annotation, Visualization and Integrated Discovery. A protein-protein interaction (PPI) network of the identified DEGs was constructed using the Search Tool for the Retrieval of Interacting Gene, and hub genes were identified. ONCOMINE and The Cancer Genome Atlas (TCGA) databases were used to verify the expression of the hub genes in oligodendroglial tumor tissues and the hub genes on the overall survival of oligodendroglial tumor patients.

Results: A total of 128 DEGs were identified from the three expression profiles. These DEGs were enriched with functional processes and pathways related to oligodendroglial tumor pathogenesis. From the PPI network, five hub genes were identified. The expression of the five hub genes was all upregulated in oligodendroglial tumor tissues compared with the control tissues. Kaplan-Meier survival curves indicated that high expression of cullin 3 (CUL3), cop9 signalosome subunit 8 (COPS8), cullin associated and neddylation dissociated 1 (CAND1), F-box protein 22 (FBXO22), and leucine rich repeat containing 41 (LRRC41) predicted poor overall survival in oligodendroglial tumor patients (all log-rank $P<0.01$ ).

Conclusions: These results revealed that the DEGs may serve as candidate key genes during oligodendroglial tumor pathogenesis. The five hub genes, including CUL3, COPS8, CAND1, FBXO22, and LRRC41, may serve as promising prognostic biomarkers in oligodendroglial tumor.

\section{Background}

Gliomas are the most common malignant brain tumors with high recurrence and lethality rates [1]. According to histological characteristics, gliomas can be classified into grades $\mathbb{\nabla}-\mathbb{Q}$ based on World Health Organization (WHO) criteria published in 2007 and 2016 [2,3]. Oligodendroglial tumor, a subtype of low-

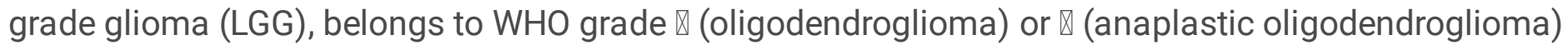
[4].Despite advances in various therapeutic strategies, such as surgery, chemotherapy, radiotherapy, immunotherapy and biologics, the prognosis and outcomes remain poor in patients suffering from oligodendroglial tumor [5]. Therefore, the identification of efficient pathological biomarkers and therapeutic targets is urgent.

It is generally considered that tumorigenesis is a complex process with a wide spectrum of genetic alterations [6]. These genes typically exhibit aberrant expression patterns and have clinical significance in cancer diagnosis and prognosis [7]. Currently, some molecules have been recognized as diagnostic and 
prognostic biomarkers in oligodendroglial tumor. For example, isocitrate dehydrogenase (IDH) mutant and $1 p / 19 q$ co-deleted oligodendroglioma is a smaller category with better prognosis; while IDH wild-type LGG has a much poorer prognosis [8]. However, the available biomarkers are not suitable for all the oligodendroglial tumor cases due to the limitations of sensitivity and specificity. Accordingly, the identification of novel functional genes may contribute to the understanding of tumor pathogenesis and the improvement of diagnosis and prognosis of oligodendroglial tumor.

In recent research, differentially expressed genes (DEGs) in tumor samples compared with normal samples can be identified using gene expression profiling arrays $[9,10]$. Some key molecules have also been reported in oligodendroglial tumor using bioinformatics analysis [11-13]. However, the number of the identified functional genes is far from sufficient to explain the mechanisms underlying the pathogenesis of oligodendroglial tumor. Thus, this study used bioinformatics analyses to further identify key genes in oligodendroglial tumor progression through three gene expression profiles from the Gene Expression Omnibus (GEO) database and assessed the clinical significance of the DEGs in oligodendroglial tumor prognosis. The expression and prognostic value of the identified key genes were further verified using the data from The Cancer Genome Atlas (TCGA) database. At last, we confirm the hub gene expression with western-blot experiment.

\section{Methods}

\section{Data collection}

In this study, we firstly downloaded three gene expression profiles from GEO database (http://www.ncbi.nlm.nih.gov/geo), including GSE15824, GSE29796, and GSE108474. The inclusion criteria for the expression profiles were as follows: (1) the samples detected are tissues, (2) all tissues are diagnosed with oligodendroglioma tissues, anaplastic oligodendroglioma tissues or normal tissues, (3) gene expression profiling of mRNA, (4) samples collected from the same racial population, (4) probes can be converted into the corresponding gene symbols, and (5) complete information for our analyses. The array data of GSE15824 included 7 oligodendroglial tumor tissues and 5 normal tissues. GSE29796 contained 72 samples, including 52 oligodendroglial tumor tissues and 20 normal tissues. GSE108474 was comprised of 67 oligodendroglial tumor tissues and 28 normal tissues.

\section{Data processing}

The DEGs between the oligodendroglial tumor samples and normal samples were analyzed by GEO2R (http://www.ncbi.nlm.nih.gov/geo/geo2r), which is a built-in online tool of GEO [14]. Adjusted $P$-value and |log fold changel ( $\mid \log \mathrm{FCl})$ were used to evaluate the significance of DEGs, and adjusted $P<0.01$ and $\| \log$ $\mathrm{FCl}>1$ were set as the cutoff criteria.

\section{Pathway and process enrichment analysis}


Metascape (https://metascape.org/gp/index.html\#/main/step1) is an essential program for the comprehensive gene function analysis, which aids the researchers to understand the biological signification of abundant genes [15]. Pathway and process enrichment analysis has been carried out with the following ontology sources: KEGG Pathway, GO Biological Processes, Reactome Gene Sets, Canonical Pathways and CORUM. All genes in the genome have been used as the enrichment background. Terms with a $P<0.01$, a minimum count of 3 , and an enrichment factor $>1.5$. Kappa scores are used as the similarity metric when performing hierachical clustering on the enriched terms, and sub-trees with a similarity of $>0.3$ are considered a cluster. The most statistically significant term within a cluster is chosen to represent the cluster.

\section{PPI network construction and module selection}

Since the interactions between proteins represent the pivotal events during cellular biological processes, we constructed a protein-protein interaction (PPI) network of the identified DEGs using the Search Tool for the Retrieval of Interacting Gene (STRING, http://string.embl.de/) database [16]. The PPI network was visualized using Cytoscape (version 3.7.1) [17], and a confidence score $\geq 0.7$ was used as the cutoff criterion. Subsequently, the modules of the PPI network were screened by the Molecular Complex Detection (MCODE) with the following parameters: degree cutoff $=2$, node score cutoff $=0.2, \mathrm{k}$-core $=2$, and maximum depth $=100$ [18].

\section{Expression Analysis}

The mRNA expression levels of the hub genes between oligodendroglial tumor tissues and normal controls were analyzed using the ONCOMINE (http://www.oncomine.org) database [19], and the data were collected from three literatures [20-22]. Terms with a similarity $>0.3$ are connected by edges. Terms with the best $P$-values from each of the 20 clusters, with the constraint that there are no more than 15 terms per cluster. The network is visualized using Cytoscape, where each node represents an enriched term and is colored first by its cluster ID and then by its $P$-value.

\section{Verification of expression and prognostic value of DEGs using TCGA data}

To confirm the clinical significance of the 5 hub genes in the prognosis of oligodendroglial tumor, data from TCGA database were further assessed using the Gene Expression Profiling Interactive Analysis (GEPIA) Øhttp://gepia.cancer-pku.cn/囚, which is a web-based tool to deliver fast and customizable functionalities based on TCGA data [20]. The expression patterns in oligodendroglial tumor tissues and the Kaplan-Meier survival curves were all performed using the GEPIA. 


\section{Human tissue specimens}

Oligodendroglial tissues, including oligodendroglioma and anaplastic oligodendroglioma, and normal brain tissues were obtained from patients undergoing surgery at Harbin Medical University Cancer Hospital, China. Collected tissues were immediately snap-frozen and stored at $-80^{\circ} \mathrm{C}$. Informed consent was obtained from each patient to approve the use of their tissues for research purposes.

\section{Clinical sample collection and western-blot analysis}

Eight cancer tissues from the patients diagnosed with oligodendroglial tumor (five oligodendroglioma WHOII, three anaplastic oligodendroglioma WHOIII) were collected for this study. All tissue samples were pathologically confirmed and immediately snap-frozen in liquid nitrogen. All procedures were conducted in accordance with the principles outlined in the Declaration of Helsinki. The study protocol was approved by the Ethics Committee of Harbin Medical University (Harbin, China).

Whole cell extracts were prepared with a cell lysis reagent (Sigma-Aldrich, St. Louis, MO, USA) according to the manual, and then, the protein was quantified by a BCA assay (Pierce, Rockford, IL, USA). The protein samples were then separated by SDS-PAGE $(10 \%)$ and detected by western blot, using polyclonal (rabbit) antibody (Santa Cruz Bio-technology, Santa Cruz, CA, USA). Goat anti-rabbit IgG (Pierce, Rockford, IL, USA) secondary antibody conjugated to horseradish peroxidase and ECL detection systems (SuperSignal West Femto, Pierce) were used for detection.

\section{Statistical analysis}

Data are expressed as mean \pm SD and analyzed by Student's $t$ test. Compared with respective controls, $P$ values $<0.05$ were considered statistically significant.

\section{Results}

\section{Identification of DEGs in oligodendroglial tumor}

According to the GEO2R analysis, a total of 880,432 , and 849 DEGs were, respectively, identified in GSE15824, GSE29796 and GSE108474. Among these DEGs, 128 genes with significant aberrant expression were extracted from all the three datasets (Figure 1), including 121 upregulated genes and 6 downregulated genes (Table 1). 


\begin{tabular}{|c|c|}
\hline DEGs & Gene name \\
\hline Upregulated & $\begin{array}{l}\text { APOC1, ELOVL6, ADD2, AGPAT5, BPTF, PNPLA8, ETV1, KRR1, TSC22D2, } \\
\text { NFIB, BTG1, TMEM97, BCLAF1, HINT11, ATP6V0A2, CYLD, UBE2Z, ZBTB20, } \\
\text { ZNF26, ID4, GTPBP8, MICALL1, LAMTOR3, LUC, L3, WASL, NCAM1, SCD, } \\
\text { LRRN3, NCBP3, SYBU, LSAMP, SQLE, MYO5A, SETD5, MRPS14, STMN3, } \\
\text { INSIG1, PNISR, EIF3F, CCSER2, MTHFD2L, COPS8, MDM2ELL2, APOE, } \\
\text { HNRNPU, RIC1, USP3, PCDHB9, PCDHB10, CSNK1A1, SUB1, PCDHB9, } \\
\text { PIK3CA, LOC101930416, LOC101929792, LOC100996724, PDE4DIP, } \\
\text { GPR173, IGF1R, ERAP1, GAP43, NMNAT2, PCNX2, NCK1, MIR4707, HAUS4, } \\
\text { MREG, SMKR1, ZNF609, MFN1, B4GALT5, NF2, PCNX1, UBASH3B, } \\
\text { CAMSAP2, TMED3, TPGS2, ASRGL1, MARK1, CADPS, PTPN14, EXOSC1, } \\
\text { SNX5, PTHLH, GSE1, RNF24, CAND1, LOC101928269, LOC100506403, } \\
\text { RUNX1, FGFR1OP2, FBXO22, CPSF6, PI4K2A, HIST2H2AA4, HIST2H2AA3, } \\
\text { TXNL4B, VRK3, SMOX, ENAH, ZFX, YWHAZ, CCDC93, QRSL1, DCAF4, } \\
\text { EPHB2, NRP2, SLC35F5, DFFA, SEC111C, LRRC41, CALU, YIPF5, OBFC1, EXT1, } \\
\text { TIMM17A, CHD9, STC2, FAM162A, YIF1B, GAD1, CUL3, EPS15L1, UCHL1, } \\
\text { UBE2J1, MIAT, PDS5A, MACF1 }\end{array}$ \\
\hline Downregulated & CDC42BPA, RNF141, SPTBN1, LDB3, ZNF252P, LIMCH1 \\
\hline \multicolumn{2}{|c|}{$\begin{array}{l}128 \text { DEGs were identified from the three profile datasets, including } 121 \text { upregulated genes and } 6 \\
\text { downregulated genes in the oligodendroglial tumor tissues compared with the normal controls. The } \\
\text { bold genes are hub genes. }\end{array}$} \\
\hline
\end{tabular}

Table 1

Upregulated and downregulated DEGs.

\section{GO analysis and pathway enrichment analysis of DEGs in oligodendroglial tumor}

The potential biological function of the identified 128 DEGs was assessed using GO analysis. As shown in Table 2, these genes were mainly enriched in biological processes related to axon development and synapse organization. Moreover, the potential signaling pathways which these DEGs involved were examined using KEGG Pathway, Reactome Gene Sets, Canonical Pathways and CORUM analysis. From the results in Table 2, we found that the DEGs were mostly enriched in axon development and synapse organization. 


\begin{tabular}{|c|c|c|c|c|}
\hline Term & Description & Count & $\log 10(p)$ & $\log 10(q)$ \\
\hline GO:0061564 & Axon development & 15 & -7.24 & -2.92 \\
\hline G0:0050808 & Synapse organization & 12 & -5.91 & -2.48 \\
\hline $\begin{array}{l}\text { R-HSA- } \\
373753\end{array}$ & Nephrin family interactions & 4 & -5.28 & -2.04 \\
\hline $\begin{array}{l}\text { R-HSA- } \\
1655829\end{array}$ & $\begin{array}{l}\text { Regulation of cholesterol biosynthesis by SREBP } \\
\text { (SREBF) }\end{array}$ & 5 & -5.05 & -1.84 \\
\hline GO:0097435 & Supramolecular fiber organization & 13 & -4.39 & -1.31 \\
\hline G0:0043409 & Negative regulation of MAPK cascade & 7 & -4.35 & -1.31 \\
\hline G0:0051646 & Mitochondrion localization & 4 & -4.05 & -1.17 \\
\hline G0:0007030 & Golgi organization & 6 & -4.03 & -1.16 \\
\hline $\begin{array}{l}\text { R-HSA- } \\
1839117\end{array}$ & Signaling by cytosolic FGFR1 fusion mutants & 3 & -4 & -1.15 \\
\hline G0:0043687 & Post-translational protein modification & 9 & -3.99 & -1.15 \\
\hline GO:0048193 & Golgi vesicle transport & 9 & -3.89 & -1.13 \\
\hline G0:0044346 & Fibroblast apoptotic process & 3 & -3.62 & -0.97 \\
\hline GO:2000144 & $\begin{array}{l}\text { Positive regulation of DNA-templated } \\
\text { transcription, initiation }\end{array}$ & 3 & -3.46 & -0.88 \\
\hline G0:0043434 & Response to peptide hormone & 9 & -3.38 & -0.83 \\
\hline G0:0044257 & Cellular protein catabolic process & 12 & -3.23 & -0.74 \\
\hline G0:0031647 & Regulation of protein stability & 7 & -3.17 & -0.71 \\
\hline M14532 & ST PHOSPHOINOSITIDE 3 KINASE PATHWAY & 3 & -3.05 & -0.64 \\
\hline GO:1903827 & Regulation of cellular protein localization & 9 & -2.83 & -0.48 \\
\hline GO:0016241 & Regulation of macroautophagy & 5 & -2.73 & -0.42 \\
\hline GO:0046822 & Regulation of nucleocytoplasmic transport & 4 & -2.7 & -0.4 \\
\hline \multicolumn{5}{|c|}{$\begin{array}{l}\text { Top20 clusters with their representative enriched terms (one per cluster). "Count" is the number of } \\
\text { genes in the user-provided lists with membership in the given ontology term. "\%" is the percentage of } \\
\text { all of the user-provided genes that are found in the given ontology term (only input genes with at least } \\
\text { one ontology term annotation are included in the calculation). "Log10(p)" is the } P \text {-value in log base } \\
10 \text {. "Log10(q)" is the multi-test adjusted } P \text {-value in log base 10. }\end{array}$} \\
\hline
\end{tabular}

Table 2

Pathway and Process Enrichment Analysis of DEGs in oligodendroglial tumor 


\section{PPI network construction and significant module analysis}

The 128 identified DEGs were all filtered into the PPI network complex, which contained 78 nodes and 105 edges (Figure 4A). Furthermore, the most significant module was extracted from the PI network, containing 11 nodes and 23 edges (Figure 4B). In this module, 5 nodes with a degree $>10$ were identified as hub genes, including F-box protein 22 (FBXO22), leucine rich repeat containing 41 (LRRC41), COP9 signalosome subunit 8 (COSP8), cullin3 (CUL3) and cullin associated neddylation dissociated 1 (CAND1) (Table 3).

\begin{tabular}{|lll|}
\hline Rank & Gene name & Score \\
\hline 1 & FBXO22 & 92 \\
2 & LRRC41 & 90 \\
\hline 3 & COPS8 & 82 \\
\hline 4 & CUL3 & 73 \\
\hline 5 & CAND1 & 72 \\
\hline
\end{tabular}

Table 3

Top five hub genes in the PPI network

\section{FBX022 expression validation and prognostic value in oligodendroglial tumor}

To further confirm the expression patterns of the 5 hub genes in oligodendroglial tumor, we obtained four datasets from the ONCOMINE database to analyze the differential expression between oligodendroglial tumor tissues and normal tissues. As shown in Figure 5A-B, the expression of FBXO22 was significantly upregulated in oligodendroglial tumor tissues compared with the normal controls in each dataset (all $P<$ 0.05), and this difference was also statistically significant combined with the two datasets $(P<0.001$, Supplementary Material 1A). In addition, expression and prognostic values were verified through TCGA Data. By using the GEPIA (http://gepia.cancer-pku.cn/), the expression patterns and prognostic value of FBXO22 were verified based on the data from TCGA database. Consistent with the expression results analyzed by ONCOMINE, the expression levels of FBXO22 assessed by TCGA data were upregulated in tumor tissues compared with the normal controls $(P<0.05$, Figure 6A). Furthermore, the survival curves shown in Figure 6F indicated that high FBXO22 expression predicted poor overall survival (log-rank $P<$ 0.05).

\section{LRRC41 expression validation and prognostic value in oligodendroglial tumor}


According to the expression investigation, we observed that the expression of LRRC41 was upregulated in oligodendroglial tumor tissues compared with normal tissues (all $P=0.045$, Figure $5 \mathrm{C}$ ). Furthermore, the expression patterns and prognostic value of LRRC41 were verified based on the data from TCGA database by using the GEPIA. Consistent with the expression results analyzed by ONCOMINE, the expression levels of LRRC41 assessed by TCGA data were upregulated in tumor tissues compared with the normal controls $(P<0.05$, Figure 6B). The survival analysis for patients revealed that the high LRRC41 expression level was associated with shorter survival time compared with the low LRRC41 expression level in oligodendroglial tumor patients (log-rank $P=3 e-4$, Figure 6G).

\section{COPS8 expression validation and prognostic value in oligodendroglial tumor}

The expression patterns of COPS8 in oligodendroglial tumor tissues were greater than those in the normal control tissues (all $P<0.05$, Figure 5D, 5E and Supplementary Material 1B). Additionally, the effect of COPS 8 on the data from TCGA database by using the GEPIA were also assessed. The results indicated that the expression levels of COPS8 assessed by TCGA data were upregulated in tumor tissues compared with the normal controls $(P<0.05$, Figure $6 \mathrm{C})$. High expression of COPS8 was also associated with shorter survival time (log-rank $P=3.2 \mathrm{e}-5$, Figure $6 \mathrm{H}$ ).

\section{CUL3 expression validation and prognostic value in oligodendroglial tumor}

The expression of CUL3 was analyzed using the ONCOMINE database was proved to be upregulated in oligodendroglial tumor tissues compared with the control tissues (All $P=0.003$, Figures $5 F$ ). We then explore CUL3 on the data from TCGA database by using the GEPIA on oligodendroglial tumors, and survival analysis was performed for patients. The results indicated that CUL3 were upregulated in tumor tissues compared with the normal controls $(P<0.05$, Figure 6D) and the high CUL3 expression level was associated with shorter survival time compared with the low CUL3 expression level in oligodendroglial tumor patients (log-rank $P=0.000031$, Figure 61$)$.

\section{CAND1 expression validation and prognostic value in oligodendroglial tumor}

The expression of CAND1 was investigated using the ONCOMINE database, and the expression level was observed to be elevated in both oligodendroglial tumor tissues compared with the normal control tissues (all $P<0.05$, Figure 5G-5I and Supplementary Material 1C). Additionally, the effect of CAND1 on the data from TCGA database were also assessed by GEPIA. The results indicated that the expression levels of CAND1 assessed by TCGA data were upregulated in tumor tissues compared with the normal controls $(P$ 
$<0.05$, Figure 6E). However, no different survival times were observed between patients with high CAND1 expression levels and patients with low CAND1 expression levels (log-rank $P=0.35$, Figure 6G).

\section{Expression of FBX022, LRRC41, COPS8, CUL3 and CAND1 in patient tissues}

Western blot assay was done applying four glioma tissues and paired non-tumor tissues (Figure 7A). Western blot analysis showed that expression of FBX022, LRRC41, COPS8, CUL3 and CAND1 were all higher in oligodendroglial tumor, in comparison with normal human brain tissues $(P<0.05)$. In addition, the five type protein expression levels in anaplastic oligodendroglioma (WHO $\otimes)$ were higher than oligodendroglioma (WHO 『). Collectively, our results suggest that FBXO22, LRRC41, COPS8, CUL3 and CAND1 were all upregulated in oligodendroglial tumor (WHO ख-WHO $\mathbb{Q})(P<0.05$, Figure 7B).

\section{Discussion}

Accurate diagnosis and prognosis remain the great challenges for the improvement of oligodendroglial tumor outcomes. To meet the clinical requirements of oligodendroglial tumor treatment, various therapeutic methods have been developed in recent decades [5,23]. Moreover, targeted therapy, which is mainly dependent on genes that have pivotal roles during tumor pathogenesis, has attracted increasing attention [24]. For example, researchers have found that the presence of telomerase reverse transcriptase (TERT) promoter mutation, Recombinant Human H3 Histone Family $3 \mathrm{~A}(\mathrm{H} 3 \mathrm{~F} 3 \mathrm{~A})$ mutation and epidermal growth factor receptor (EGFR) amplification showed negative prognostic impacts in IDH-wild type LGG [25]. To identify novel key genes that might be involved in oligodendroglial tumor pathogenesis, we performed a systematic analysis of three expression profiles from GEO database using bioinformatics analysis. The DEGs in the expression profiles and the prognostic value of the key genes were assessed in the present study.

A total of 126 oligodendroglial tumor samples and 53 normal control samples were included in the three expression profiles, and 128 DEGs were screened for further analyses. According to the pathway and process enrichment analysis, the identified DEGs were shown to be enriched in biological processes that related to axon development and synapse organization. It is generally considered that cortical axon outgrowth and synapse organization is closely related with neurodegenerative disorders, brain ischemia and cancer [26].

The PPI network of the DEGs was constructed, and five hub genes were extracted from a significant module, including FBOX22, LRRC41, COPS8, CUL3 and CAND1. A study by Liang $Y$ et al [27] also focused on the DEGs in oligodendroglial tumor tissues compared with the normal controls, and CUL3 was identified as the hub genes, which was consistent with our corresponding data. Furthermore, the expression patterns of the five hub genes were found all upregulated in oligodendroglial tumor tissues compared with the normal controls, and their prognostic value was evaluated by plotting the Kaplan- 
Meier survival curves. The analysis results indicated that the expression levels of FBX022, LRRC41, COPS8 and CUL3 were associated with the overall survival of oligodendroglial tumor patients except CAND1, which might be due to the limited sample size and the incomplete survival information. Additionally, Western blot analysis showed that the protein levels of FBXO22, LRRC41, CUL3, CAND1 and COPS8 were significantly increased in the oligodendroglial tumor tissues compared with normal tissues. Generally, results indicate that these genes might serve as promising prognostic biomarkers in oligodendroglial tumor.

The F-box protein FBXO22, a not well-recognized protein, contains the 40-amino-acid F-box domain, which binds Skp-1 to create a link to Cullin-1, then form the Skp-1, Cullin-1, F-box protein (SCF) complex to constitute an E3 ligase for ubiquitylation [28]. Zhu XN et al [29] finding show that FBXO22 is highly expressed in human lung cancer at the protein level. Bai $\mathrm{J}$ et al [30] reveal that SCFFBXO22 targets HDM2 for degradation and possesses inhibitory effects against breast cancer tumor cell invasion and metastasis. We also found the increased FBXO22 expression in oligodendroglial tumor samples and proved its prognostic value for cancer patients. The molecular mechanisms underlying the role of FBXO22 in human cancers await more research.

LRRC41 protein is involved in the pathway protein ubiquitination, which is part of Protein modification. In recent years, a number of studies reported that LRRC super family members are up-regulated in cancer cells [31]. Our study also showed the upregulated expression of LRRC41 in oligodendroglial tumor tissues and reported their prognostic value for the patients. At the same time, the clinical significance verification using TCGA data showed that the difference between the survival distributes of low and high LRRC41 expression groups was statistically significant, while Western blot analysis showed the same outcome. Collectively, the role of LRRC41 in cancer pathogenesis and the related molecular mechanisms need to be assessed with in-depth studies.

The COP9 signalosome (CSN) consisting of 8 unique protein subunits (COPS1 through COPS8) serves as the cullin deneddylase, regulating the catalytic dynamics of cullin RING ligases (CRLs), the largest family of ubiquitin ligases [32]. At the same time, some findings reported obviously challenge this theory. Hence, they propose that COPS8/CSN regulates the SR protein levels and thereby the activity of CRLs through both transcriptional and post-translational mechanisms. We also found the over expression of COPS8 in oligodendroglial tumor. The mechanism of COPS8 in cancer is still unclear.

CUL3 were the key hub genes identified in the astrocytoma relative to the oligodendroglial tumor. CUL3 mediates neurofibromin destabilization, which underlies glioblastoma pathogenesis [33]. CUL3 is also critical to the full activation of the Ras/ERK pathway, which is essential for cell proliferation, arrest, differentiation, survival, and apoptosis [34]. This study showed elevated CUL3 expression levels in oligodendroglial tumor tissues and demonstrated its potential as a prognostic biomarker in this malignancy. The upregulation of CUL3 and its prognostic value have been reported in LGG in previous studies, which also revealed its correlation with tumor onset [35]. Further studies should be carried out to explore the mechanisms underlying the role of CUL3 during cancer pathogenesis. 
Cand1 is a Cul1 associated protein whose binding is mutually exclusive with the F-box Protein-Skp1 subcomplex and is also blocked by attachment of the ubiquitin-like protein Nedd8 to lysine 720 of Cul [36,37]. Zhihui Che et al [38] pioneered in reporting high CAND1 expression as a predictor of poor prognosis for hepatocellular carcinoma (HCC) patients. CAND1 silencing suppressed HCC cell proliferation by inducing caspase-8/RIP1-dependent apoptosis. At present, we found the increased CAND1 expression in oligodendroglial tumor samples and proved its prognostic value for cancer patients. The molecular mechanisms underlying the role of CAND1 in human cancers await more research.

\section{Conclusions}

In conclusion, this study identified 128 DEGs from three expression profiles by integrated bioinformatics analysis. These DEGs may contain key genes involved in oligodendroglial tumor pathogenesis. In addition, FBX022, LRRC41, COPS8, CUL3 and CAND1 were the top five hub genes and serve as candidate prognostic biomarkers in oligodendroglial tumor. The results of this study further enrich the number of key genes that may be involved in the pathogenesis of oligodendroglial tumor and give in silico evidence for the key genes in the prognosis of oligodendroglial tumor. However, the number of samples included in this study was relatively small. Experimental verification and additional studies with larger sample sizes are required to confirm the present results.

\section{List Of Abbreviations}

DEGs, differentially expressed genes

PPI, protein-protein interaction

LGG, low-grade glioma

\section{Declaration}

\section{Consent for publication}

Not applicable.

\section{Availability of data and materials}

The data used to support the fndings of this study are available from the corresponds author upon request

\section{Competing interests}

The authors declare that there is no confict of interests regarding the publication of this paper. 


\section{Authors' contributions}

$\mathrm{ZHL}, \mathrm{HBB}$ and PL conceived and designed the study, and the experiments were performed by ZHL and HBB. ZHL, HBB and PL analyzed the data and wrote the manuscript. All authors read and approved the final manuscript.

\section{Ethics approval and consent to participate}

Not applicable.

\section{Funding}

Not applicable.

\section{Achnowledgements}

Not applicable.

\section{References}

1. Ostrom QT, Gittleman H, Truitt G, Boscia A, Kruchko C, Barnholtz-Sloan JS. CBTRUS Statistical Report: Primary Brain and Other Central Nervous System Tumors Diagnosed in the United States in 2011-2015. Neuro Oncol. 2018;20(suppl_4):iv1-iv86.

2. Louis DN, Ohgaki H, Wiestler OD, et al. The 2007 WHO classification of tumours of the central nervous system. Acta Neuropathol. 2007;114(2):97-109.

3. Louis DN, Perry A, Reifenberger G, et al. The 2016 World Health Organization Classification of Tumors of the Central Nervous System: a summary. Acta Neuropathol. 2016;131(6):803-20.

4. Choi J, Kim SH, Ahn SS, et al. Extent of resection and molecular pathologic subtype are potent prognostic factors of adult WHO grade II glioma. Sci Rep. 2020;10(1):2086.

5. Dhawan S, Patil CG, Chen C, Venteicher AS. Early versus delayed postoperative radiotherapy for treatment of low-grade gliomas. Cochrane Database Syst Rev. 2020;1:CD009229.

6. Nguyen LH, Goel A, Chung DC. Pathways of Colorectal Carcinogenesis. Gastroenterology. 2020;158(2):291-302.

7. Xie FJ, Lu HY, Zheng QQ, et al. The clinical pathological characteristics and prognosis of FGFR1 gene amplification in non-small-cell lung cancer: a meta-analysis. Onco Targets Ther. 2016;9:171-81.

8. Molinari E, Curran OE, Grant R. Clinical importance of molecular markers of adult diffuse glioma. Pract Neurol. 2019;19(5):412-416. 
9. Long H, Liang C, Zhang X, et al. Prediction and Analysis of Key Genes in Glioblastoma Based on Bioinformatics. Biomed Res Int. 2017;2017:7653101.

10. Wang M, Wang L, Wu S, Zhou D, Wang X. Identification of Key Genes and Prognostic Value Analysis in Hepatocellular Carcinoma by Integrated Bioinformatics Analysis. Int J Genomics. 2019;2019:3518378.

11. Wang L, Zhao K, Ren B, et al. Expression of cortactin in human gliomas and its effect on migration and invasion of glioma cells. Oncol Rep. 2015;34(4):1815-24.

12. Shi T, Chen J, Li J, Yang BY, Zhang QL. Identification of key gene modules and pathways of human glioma through coexpression network. J Cell Physiol. 2019;234(2):1862-1870.

13. Bo L, Wei B, Wang Z, Kong D, Gao Z, Miao Z. Identification of key genes in glioma CpG island methylator phenotype via network analysis of gene expression data. Mol Med Rep. 2017;16(6):95039511.

14. Barrett T, Wilhite SE, Ledoux P, et al. NCBI GEO: archive for functional genomics data sets-update. Nucleic Acids Res. 2013;41(Database issue):D991-5.

15. Lu Y, Zhang J, Li L, Li S, Yang Z. Carcinogenesis effects of E2F transcription factor 8 (E2F8) in hepatocellular carcinoma outcomes: an integrated bioinformatic report. Biosci Rep. 2020;40(2).

16. Szklarczyk D, Franceschini A, Wyder S, et al. STRING v10: protein-protein interaction networks, integrated over the tree of life. Nucleic Acids Res. 2015;43(Database issue):D447-52.

17. Zhang C, Zheng Y, Li X, Hu X, Qi F, Luo J. Genome-wide mutation profiling and related risk signature for prognosis of papillary renal cell carcinoma. Ann Transl Med. 2019;7(18):427.

18. Bader GD, Hogue CW. An automated method for finding molecular complexes in large protein interaction networks. BMC Bioinformatics. 2003;4:2.

19. Rhodes DR, Yu J, Shanker K, et al. ONCOMINE: a cancer microarray database and integrated datamining platform. Neoplasia. 2004;6(1):1-6.

20. Darlix A, Zouaoui S, Virion JM, et al. Significant heterogeneity in the geographical distribution of diffuse grade II/III gliomas in France. J Neurooncol. 2014;120(3):547-55.

21. Wang $H$, Feng $Y, B a o Z$, et al. Epigenetic silencing of KAZALD1 confers a better prognosis and is associated with malignant transformation/progression in glioma. Oncol Rep. 2013;30(5):2089-96.

22. Kouwenhoven MC, Kros JM, French PJ, et al. 1p/19q loss within oligodendroglioma is predictive for response to first line temozolomide but not to salvage treatment. Eur J Cancer. 2006;42(15):2499503.

23. Tang Z, Li C, Kang B, Gao G, Li C, Zhang Z. GEPIA: a web server for cancer and normal gene expression profiling and interactive analyses. Nucleic Acids Res. 2017;45(W1):W98-W102.

24. Yang P, Cai J, Yan W, et al. Classification based on mutations of TERT promoter and IDH characterizes subtypes in grade II/III gliomas. Neuro Oncol. 2016;18(8):1099-108.

25. Vuong HG, Tran TTK, Ngo HTT, et al. Prognostic significance of genetic biomarkers in isocitrate dehydrogenase-wild-type lower-grade glioma: the need to further stratify this tumor entity - a meta- 
analysis. Eur J Neurol. 2019;26(3):379-387.

26. Ono Y, Saido TC, Sorimachi H. Calpain research for drug discovery: challenges and potential. Nat Rev Drug Discov. 2016;15(12):854-876.

27. Liang Y, Diehn $\mathrm{M}$, Watson $\mathrm{N}$, et al. Gene expression profiling reveals molecularly and clinically distinct subtypes of glioblastoma multiforme. Proc Natl Acad Sci U S A. 2005;102(16):5814-9.

28. Jin J, Cardozo T, Lovering RC, Elledge SJ, Pagano M, Harper JW. Systematic analysis and nomenclature of mammalian F-box proteins. Genes Dev. 2004;18(21):2573-80.

29. Zhu XN, He P, Zhang L, et al. FBXO22 mediates polyubiquitination and inactivation of LKB1 to promote lung cancer cell growth. Cell Death Dis. 2019;10(7):486.

30. Bai J, Wu K, Cao MH, et al. SCF(FBXO22) targets HDM2 for degradation and modulates breast cancer cell invasion and metastasis. Proc Natl Acad Sci U S A. 2019;116(24):11754-11763.

31. Schenkova K, Lutz J, Kopp M, Ramos S, Rivero F. MUF1/leucine-rich repeat containing 41 (LRRC41), a substrate of RhoBTB-dependent cullin 3 ubiquitin ligase complexes, is a predominantly nuclear dimeric protein. J Mol Biol. 2012;422(5):659-673.

32. Abdullah A, Eyster KM, Bjordahl T, Xiao P, Zeng E, Wang X. Murine Myocardial Transcriptome Analysis Reveals a Critical Role of COPS8 in the Gene Expression of Cullin-RING Ligase Substrate Receptors and Redox and Vesicle Trafficking Pathways. Front Physiol. 2017;8:594.

33. Hollstein PE, Cichowski K. Identifying the Ubiquitin Ligase complex that regulates the NF1 tumor suppressor and Ras. Cancer Discov. 2013;3(8):880-93.

34. Bigenzahn JW, Collu GM, Kartnig F, et al. LZTR1 is a regulator of RAS ubiquitination and signaling. Science. 2018;362(6419):1171-1177.

35. Wang S, Jin F, Fan W, et al. Gene expression meta-analysis in diffuse low-grade glioma and the corresponding histological subtypes. Sci Rep. 2017;7(1):11741.

36. Cheng W, Yin S, Tu Y, Mei H, Wang Y, Yang Y. SICAND1, encoding cullin-associated Nedd8-dissociated protein 1 , regulates plant height, flowering time, seed germination, and root architecture in tomato. Plant Mol Biol. 2020;102(4-5):537-551.

37. Yu Q, Hu Z, Shen Y, et al. Gossypol inhibits cullin neddylation by targeting SAG-CUL5 and RBX1-CUL1 complexes. Neoplasia. 2020;22(4):179-191.

38. Che Z, Liu F, Zhang W, et al. Targeting CAND1 promotes caspase-8/RIP1-dependent apoptosis in liver cancer cells. Am J Transl Res. 2018;10(5):1357-1372.

\section{Figures}




\section{GSE15824 GSE29796}

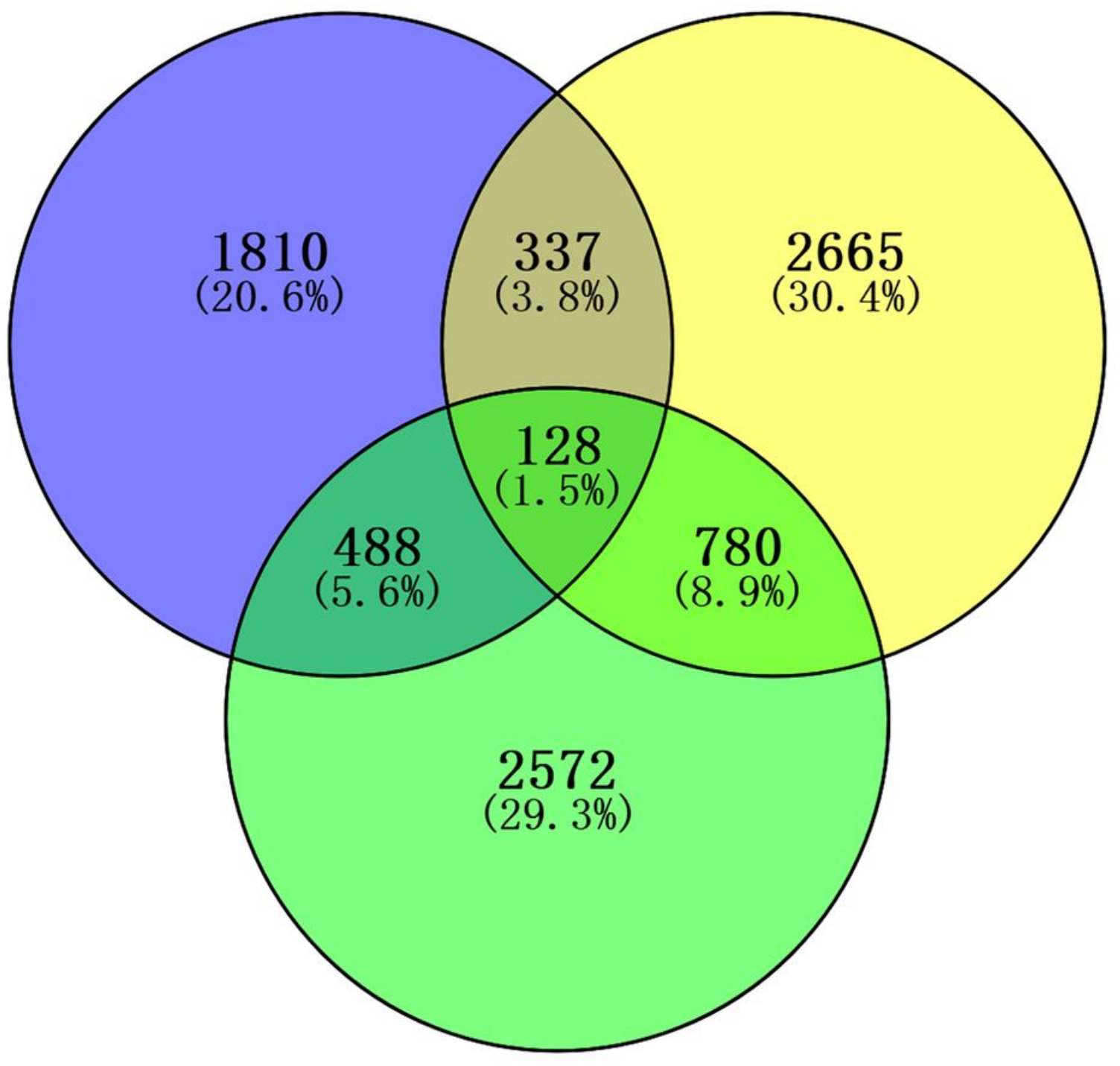

GSE108474

Figure 1

DEG identification in 3 mRNA expression profiles (GSE15824, GSE29796, and GSE108474). A total of 128 DEGs were identified from the three expression profiles. 


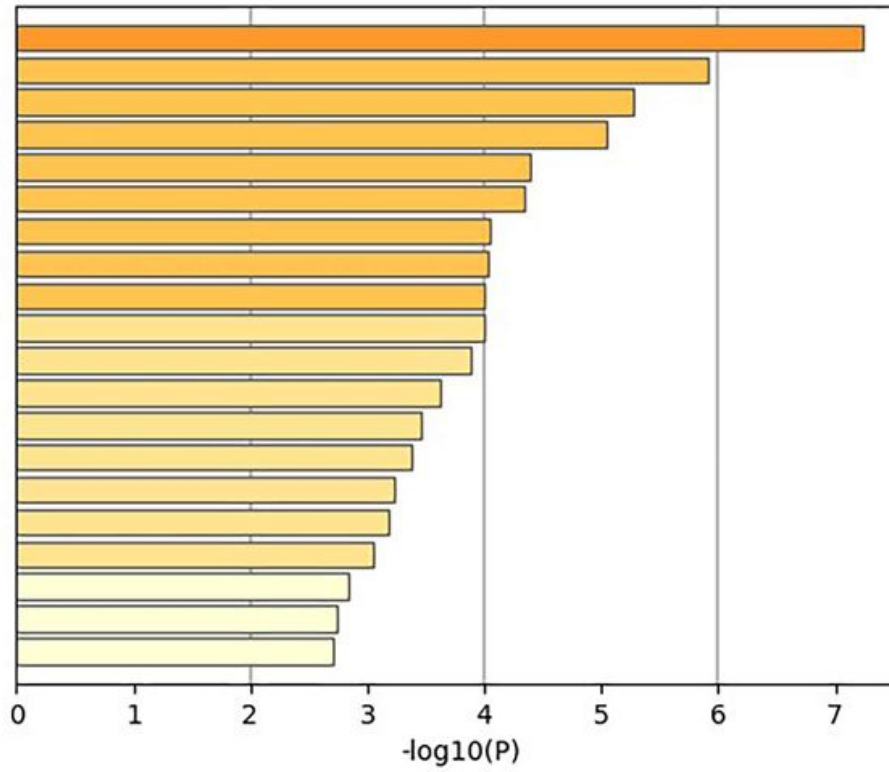

GO:0061564: axon development

GO:0050808: synapse organization

R-HSA-373753: Nephrin family interactions

R-HSA-1655829: Regulation of cholesterol biosynthesis by SREBP (SREBF)

GO:0097435: supramolecular fiber organization

GO:0043409: negative regulation of MAPK cascade

GO:0051646: mitochondrion localization

GO:0007030: Golgi organization

R-HSA-1839117: Signaling by cytosolic FGFR1 fusion mutants

GO:0043687: post-translational protein modification

GO:0048193: Golgi vesicle transport

GO:0044346: fibroblast apoptotic process

GO:2000144: positive regulation of DNA-templated transcription, initiation

GO:0043434: response to peptide hormone

GO:0044257: cellular protein catabolic process

GO:0031647: regulation of protein stability

M14532: ST PHOSPHOINOSITIDE 3 KINASE PATHWAY

GO:1903827: regulation of cellular protein localization

GO:0016241: regulation of macroautophagy

GO:0046822: regulation of nucleocytoplasmic transport

Figure 2

Bar graph of enriched terms across input gene lists, colored by P-values.
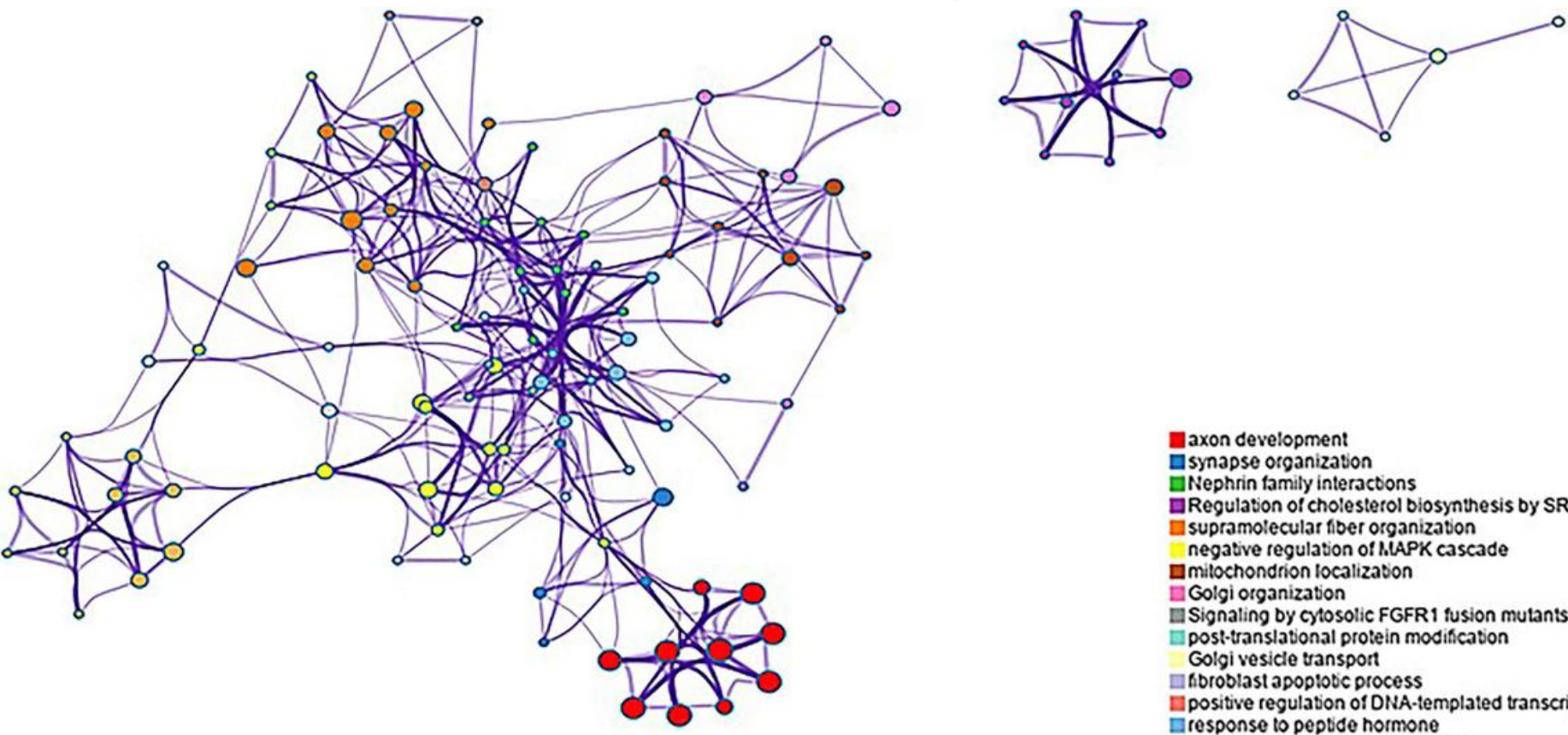

$\int_{0}^{0}$

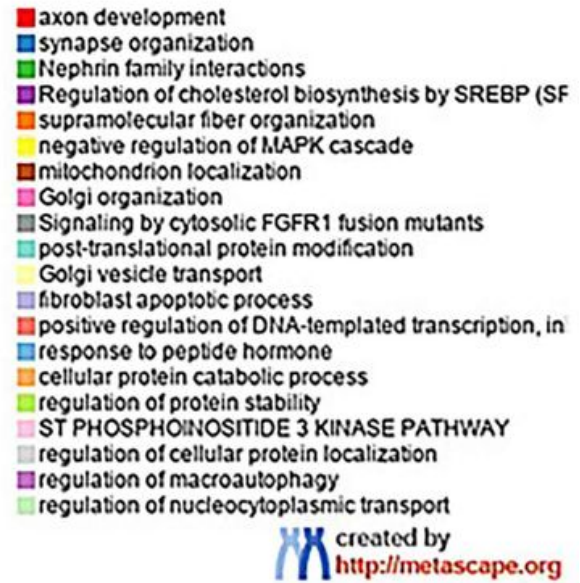

Figure 3

Further capture the relationships between the terms 


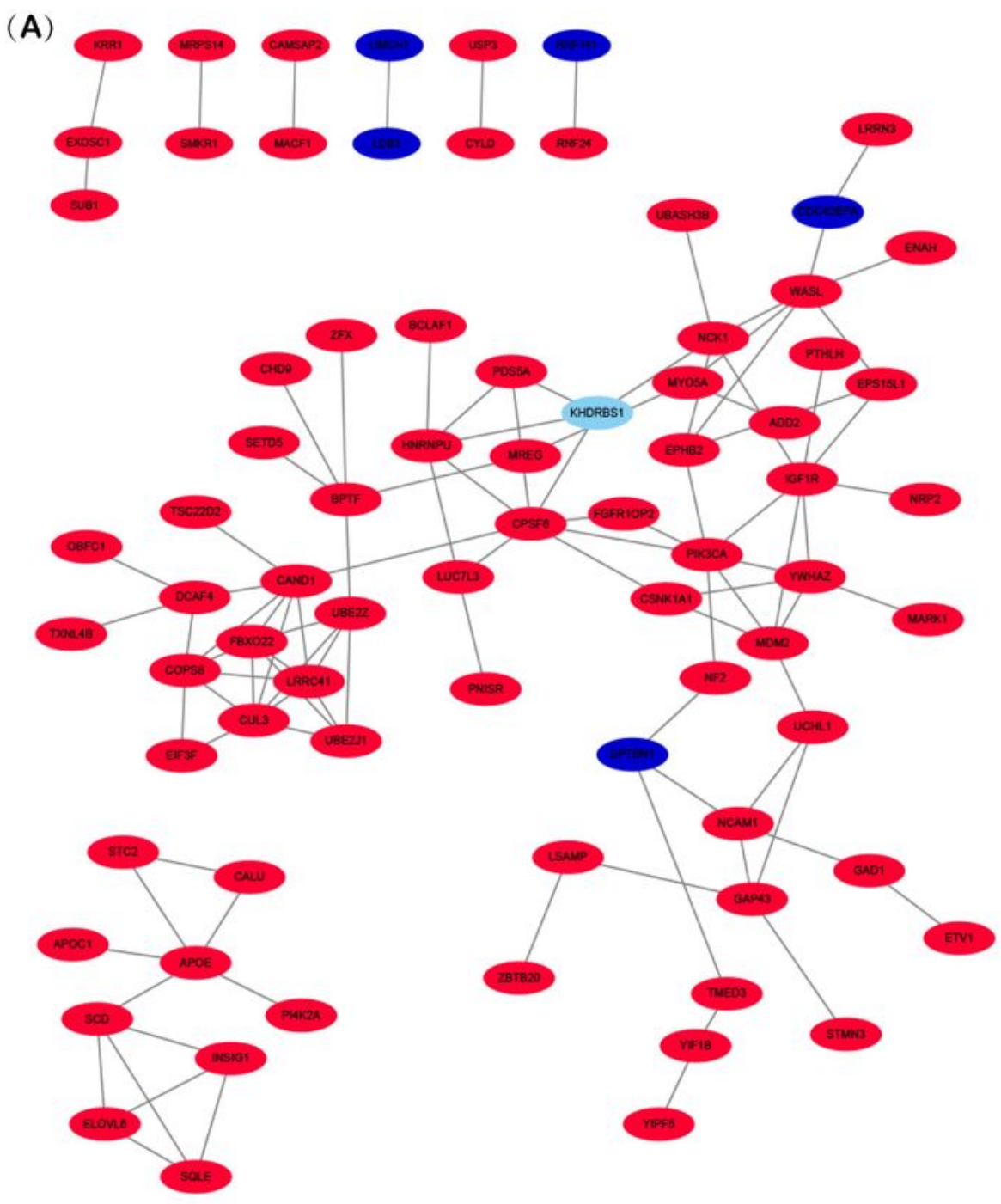

(B)

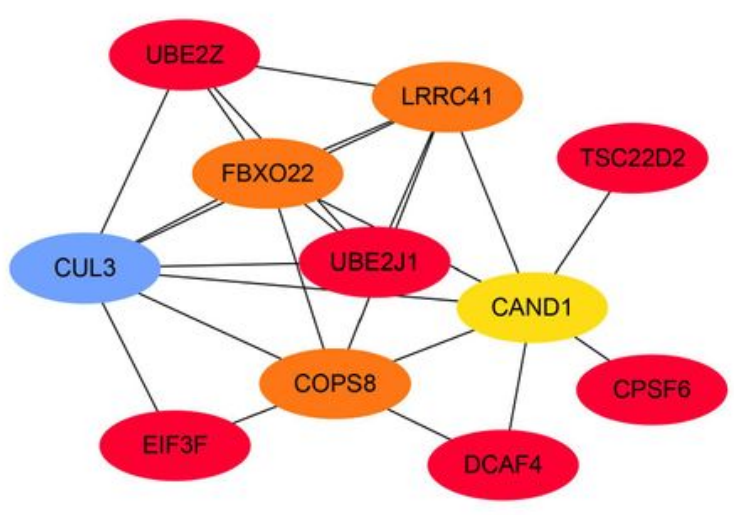

Figure 4

A PPI network of the DEGs and a significant module in the PPI network. (A) DEG PPI network contained 78 nodes and 105 edges. (B) The significant module obtained from the PPI network. The yellow, orange, and blue nodes represented top five hub genes in the network. 
(A) FBXO22 Expression in French Brain

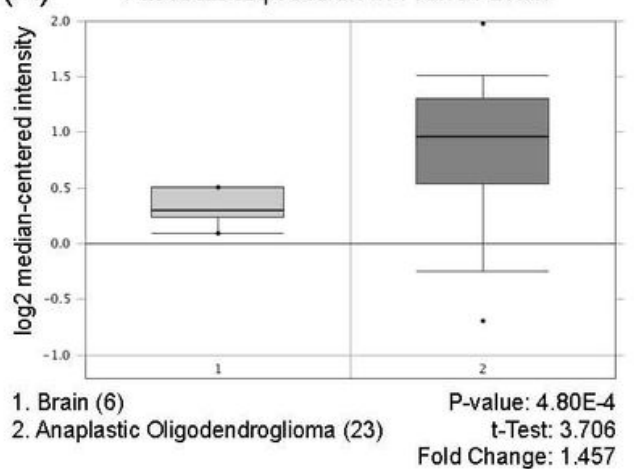

(D) COPS8 Expression in Shai Brain

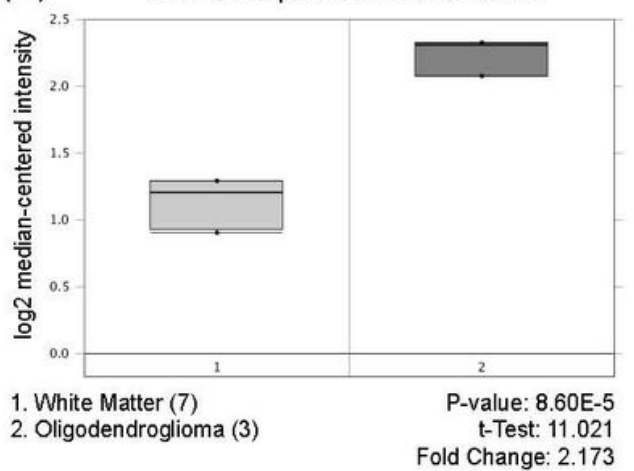

(G)

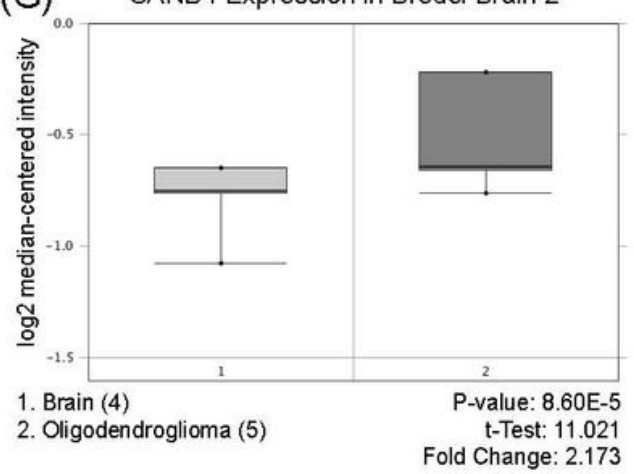

(B)

FBXO22 Expression in Sun Brain

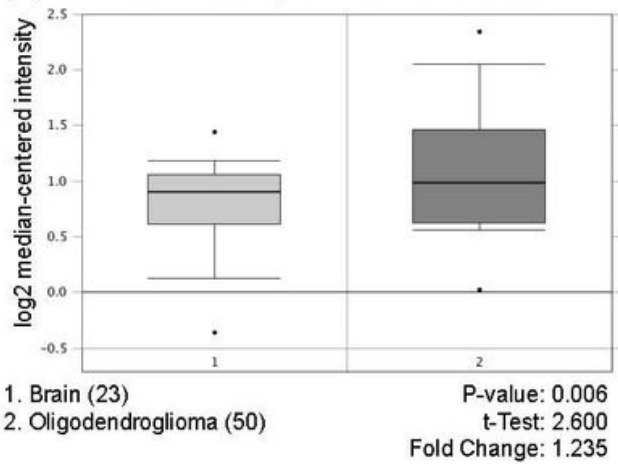

(E)

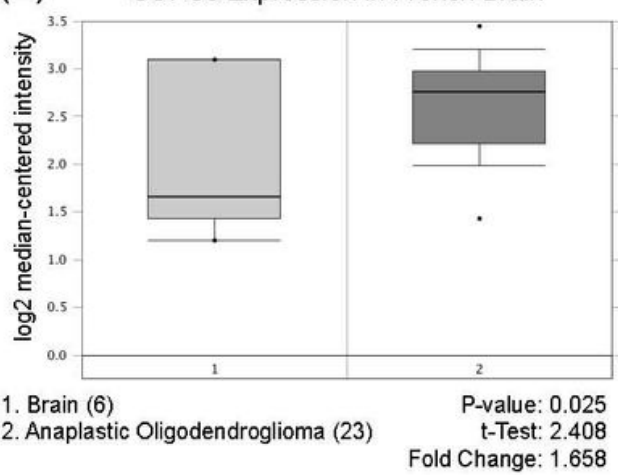

$(\mathrm{H})$

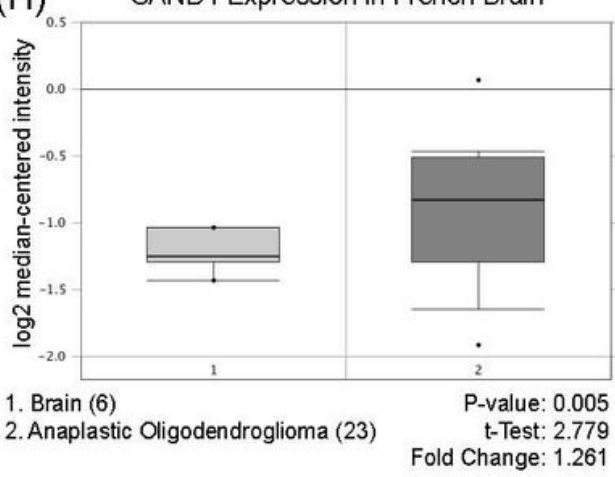

(C) LRRC41 Expression in French Brain

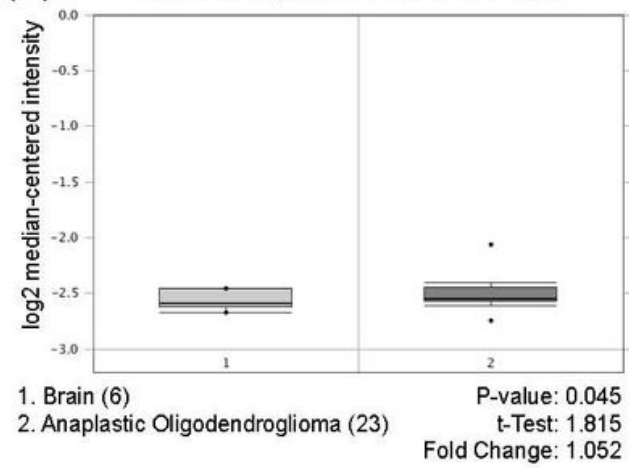

$(\mathrm{F})$

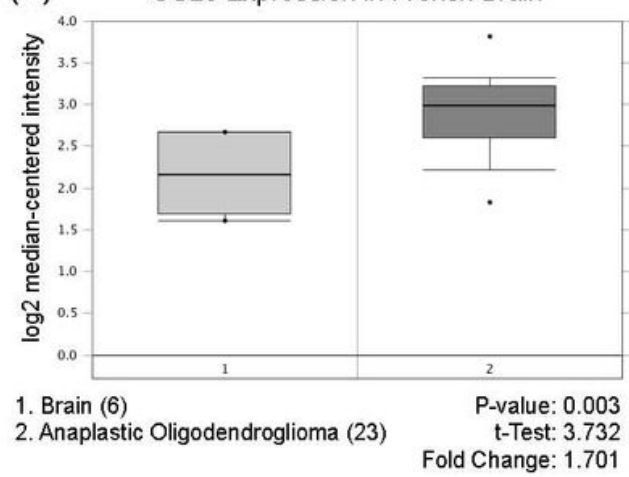

(1)

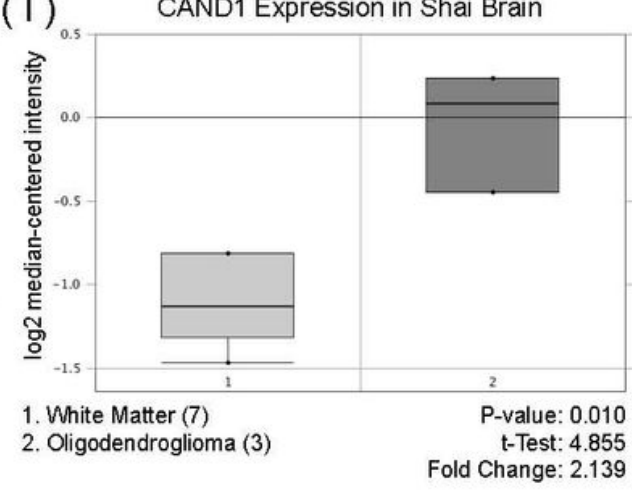

\section{Figure 5}

The expression data of five hub genes in oligodendroglial tumor collected from ONCOMINE. (A) FBXO22 Expression in French Brain; (B) FBXO22 Expression in Sun Brain; (C) LRRC41 Expression in French Brain; (D) COPS8 Expression in Shai Brain; (E) COPS8 Expression in French Brain; (F) CUL3 Expression in French Brain; (G) CAND1 Expression in Bredel Brain 2; (H) CAND1 Expression in Shai Brain; (I) CAND1 Expression in French Brain. The expression data indicated that all of five hub genes expression was upregulated inoligodendroglial tumor tissues compared with the normal control tissues (all $\mathrm{P}<0.05)$. 

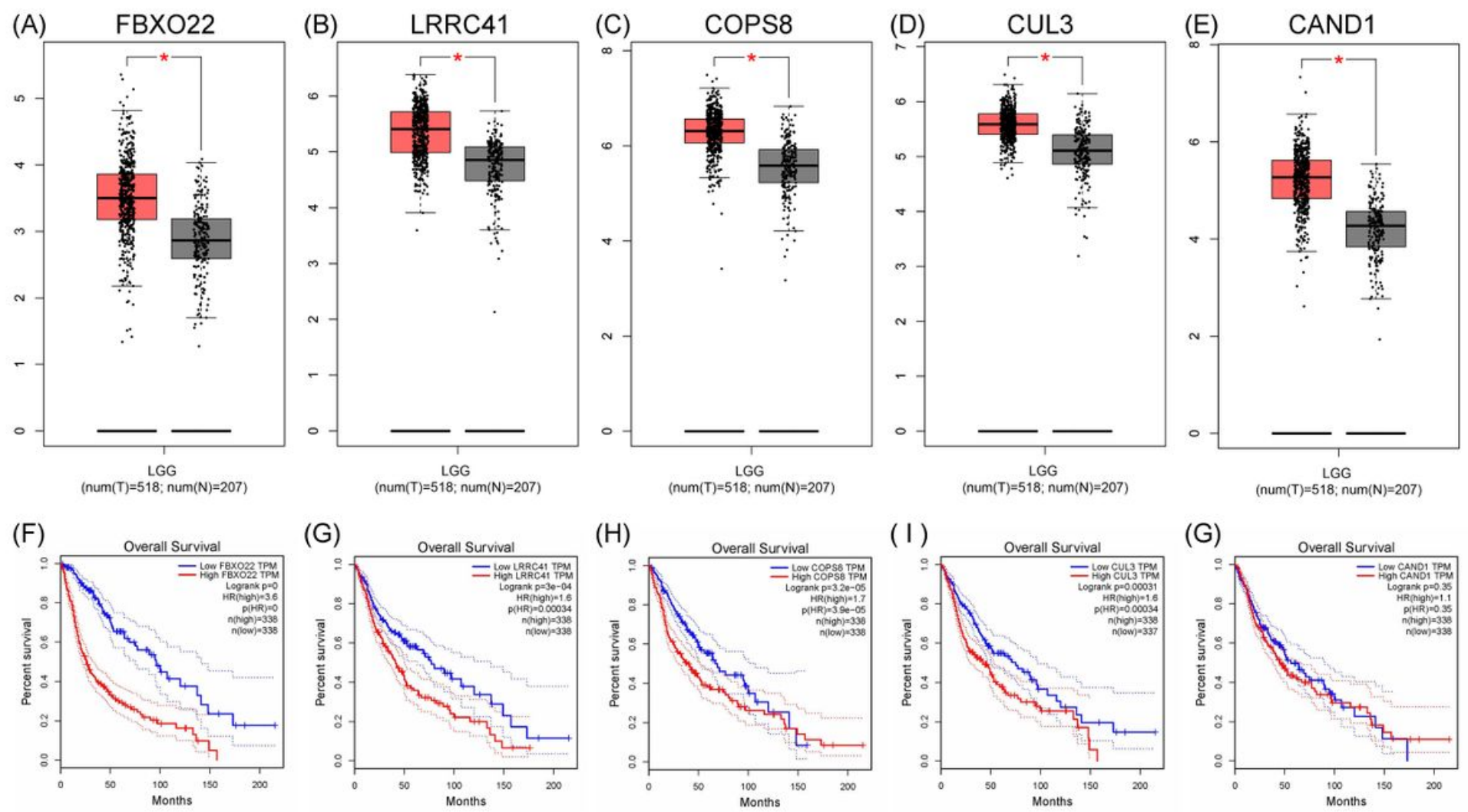

\section{Figure 6}

Expression and prognostic value verification using TCGA data. TCGA data indicated that the expression levels of FBXO22 (A), LRRC41 (B), COPS8 (C), CUL3 (D) and CAND1 (E) were all increased in tumor samples compared with normal controls (all $P<0.05)$. The patients with high FBXO22 (F), LRRC41 $(G)$, COPS8 $(\mathrm{H})$ and CUL3 (I) expression had poor overall survival compared with those with low expression of these genes. But in CAND1 (G) the difference of survival distribution between high and low expression groups was not statistically significant. T: tumor; N: normal. 


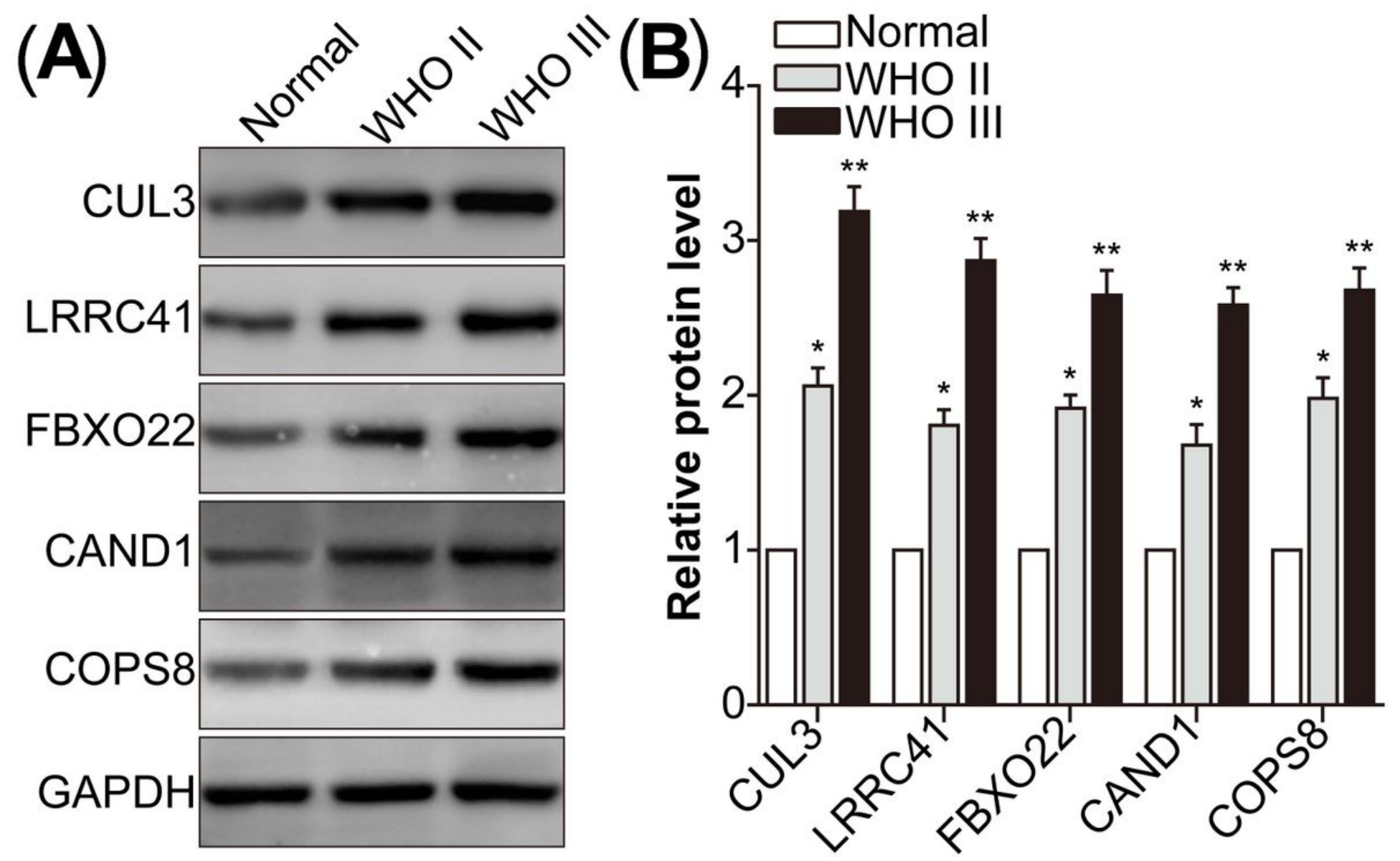

Figure 7

Western blot analysis of five hub genes in oligodendroglial tumor patient tissues. (A) CUL3, LRRC41, FBX022, CAND1 and COPS8 protein expression was determined by Western blot analysis. (B) Histogram of relative protein expression levels with relative ratios. All experiments were carried out in triplicate. Data are shown as mean $\pm \mathrm{SE}$. ${ }^{*} \mathrm{P}<0.05$ vs Control, $* \star \mathrm{P}<0.01$ vs Control.

\section{Supplementary Files}

This is a list of supplementary files associated with this preprint. Click to download.

- Supplementarymaterial.pdf 